\title{
THE MODAL STATUS OF LAWS: IN DEFENGE OF A HYBRID VIEW
}

\author{
By Tuomas E. Tahкo
}

\begin{abstract}
Three popular views regarding the modal status of the lawes of nature are discussed: Humean Supervenience, nomic necessitation, and scientific/dispositional essentialism. These viewes are examined especially with regard to their take on the apparent modal force of laws and their ability to explain that modal force. It will be suggested that none of the three viewes, at least in their strongest form, can be maintained if some lawes are metaphysically necessary, but others are metaphysically contingent. Some reasons for thinking that such variation in the modal status of laws exists will be presented with reference to physics. This drives us towards a fourth, hybrid view, according to which there are both necessary and contingent laws. The prospects for such a view are studied.
\end{abstract}

Keywords: laws, essentialism, metaphysical necessity, physical necessity, natural kinds, dispositions.

\section{INTRODUCTION}

There are three popular views regarding the modal status of the laws of nature: the Humean Supervenience (HS) approach originating in Lewis's work (e.g., Lewis I986), the nomic necessitation approach primarily familiar from Armstrong's (as well as Dretske's and Tooley's) work (e.g., Armstrong I983), and the scientific/dispositional essentialist approach defended, for instance, by Ellis (200I), Ellis \& Lierse (1994), and Bird (2007). ${ }^{1}$ All of these views come in many varieties, and there are also alternative approaches, such as Mumford's (2004) lawlessness approach, Lowe's (2006) essentialist approach, and Maudlin's (2007) primitivism about laws. The following question regarding Modal Force (MF) is central to all of these approaches:

(MF) How can one explain the apparent modal force that distinguishes genuine laws from mere regularities?

\footnotetext{
${ }^{1}$ See Beebee (2000) for a helpful comparison between the first two views.
} 
Any complete account of the metaphysics of laws should be able to explain the apparent modal force presupposed by (MF). But it is not altogether clear what would count as a satisfactory explanation. In fact, one problem in the extensive literature concerning laws derives exactly from the very different criteria that philosophers demand from a satisfactory explanation of the modal force of laws. The preliminary hypothesis of this paper is that the different criteria can all be useful - even correct - but they correspond to different types of laws. In other words, the mistake has been to search for a uniform account.

In their strongest form, each of the three popular views falls to one or the other end of the extreme regarding the modal status of laws i.e., either all laws are contingent or all laws are necessary. ${ }^{2}$ From the viewpoint of Humeanism, laws are thoroughly contingent, mere regularities that could have been different, yet 'supervene on particular matters of fact' (Beebee 2000: 572). According to scientific/dispositional essentialism, laws are metaphysically necessary, intimately related to the essential properties of the things they concern. Very roughly, this difference may be considered to concern the origin or source of laws: either they originate in the random arrangement of things or they flow from essential properties.

The nomic necessitation approach has a somewhat misleading name, as it is in fact at the contingent end of the spectrum. However, Armstrong would not be happy with either of the two mentioned options regarding the origin of laws, for he states that the idea of nomic necessitation is exactly that we can distinguish laws from mere regularities, contra Lewis (Armstrong 1997: 223). Yet, nomic necessitation does not directly concern metaphysical necessity, which is what the mentioned spectrum of the two extremes is supposed to capture. ${ }^{3}$ To be perfectly clear about this, here is a helpful passage from Bird: ${ }^{4}$

Armstrong wants to capture the idea that nomic necessitation has some kind of modal force - not the "hard" kind associated with full-on metaphysical necessity, but a "soft" kind associated with nomic modality (including explanatory force and the ability to support counterfactuals) and consistent with metaphysical contingency. (Bird 2005: 148)

${ }^{2}$ There are some caveats. For instance, Hendry and Rowbottom (2009) argue that (permissive) scientific/dispositional essentialism is compatible with interworld variation in a property's dispositional profile, hence giving room for slight variation in laws of nature. Regardless, I will focus on versions of scientific/dispositional essentialism that defend the necessity of all laws. By way of justification, Bird (2007: $48 \mathrm{ff}$.) takes it that consistent dispositional essentialists should be committed to the necessity of all laws - the alternative would be an 'untidy metaphysics, with two classes of laws' (p. 49).

${ }^{3}$ In fact, some nomic necessitation accounts (e.g., Swoyer 1982) do consider all laws to be metaphysically necessary, but the original Armstrong-Dretske-Tooley account was certainly in favour of metaphysically contingent laws.

${ }^{4}$ Some, like Hendry \& Rowbottom (2009), seem to conflate nomic necessitation and dispositional essentialism, claiming that both are committed to the metaphysical necessity of laws. As the quotation from Bird makes clear, this does not reflect Armstrong's (original) version of nomic necessitation. See also Schrenk's (201 I) discussion on Armstrong and nomic necessitation. 
Both Armstrong's nomic necessitation approach and the Humean branch reject the view that laws have modal force of the 'hard' variety. In this limited sense, we can lump them together when we examine whether a plausible account of the apparent modal force of the laws of nature is available. A comprehensive - or even fair - treatment of HS and the related Best System Analysis would certainly require a paper of its own. But since such an analysis is not central to the argument of this paper, I will instead just outline the similarities between (some versions of) HS and the nomic necessitation approach.

If we apply Lewis's (1986: ix) traditional characterization of HS to laws, we can understand the Humean view as stating that the laws of nature supervene on the totality of local matters of particular fact (Bird 2007: 82). There is a particular aspect typical to many variations of $\mathrm{HS}$ which is of special interest to us. ${ }^{5}$ This aspect is related to a view about properties called categoricalism, or, as Bird (2007: 66) calls it, categorical monism: all sparse/fundamental properties are categorical (as opposed to dispositional). The Humean approach holds that fundamental properties do not have essential causal powers, or indeed any essential features. Hence, properties are also devoid of immanent modality. More generally, categorical properties do not 'metaphysically necessitate anything about property instantiations in wholly distinct regions' (Loewer 2012: II6). Bird thinks that a commitment to categoricalism, which is shared by the Armstrong-type nomic necessitation and the Humean approach to laws, is problematic:

The categoricalist view denies that there are potencies or indeed any other kind of modality immanent in regularities - the Humean Supervenience thesis says that they merely supervene on the distribution of categorical properties among particulars (and furthermore nothing explains that distribution). (Bird 2007: 90)

According to the categoricalist view, laws are contingent regularities that concern categorical properties. Since a commitment to categoricalism is typical both for HS and the nomic necessitation view, the scientific/dispositional essentialist might hope to refute both views with the same argument against categoricalism, building on Bird (2005). Evidence in favour of immanent modality in at least some regularities should be enough. In the course of this paper, some evidence for such modal content will be presented. Hence, in partial agreement with Bird, the hypothesis of this paper is that Armstrong's 'soft' modal force cannot be enough, at least not for all laws. Given this acknowledgement, my starting point is evidently closer to the scientific/dispositional essentialist line than the HS or the nomic necessitation approach. Therefore, even though

${ }^{5}$ See Earman \& Roberts (2005a,b) for some discussion of the various formulations of HS and an attempt at and defence of a better formulation. There are no doubt better and worse formulations of HS out there, but I take it that a core corollary of HS is that there is no modality immanent in laws, that is, any modality that we associate with laws is a supervenient feature of the Humean mosaic. 
the positive argument that will be presented is not directly concerned with any of the mentioned views about laws, a type of essentialist position will be adopted for the sake of argument. The suggestion is that the truth of the matter lies somewhere between the two extremes: laws are not mere random regularities or subject only to nomic necessitation, nor are they (all) metaphysically necessary. This leaves open the possibility that some laws are contingent and some are necessary - a 'mixed' view. Such a view is explicitly denied by Bird (e.g., 2007.: $48 \mathrm{ff}$ ), who gives priority to a unified account. ${ }^{6}$ But the view to be presented is not strictly a mix of scientific/dispositional essentialism and the Humean or nomic necessitation view, but rather a fourth view distinct from the three popular views, although partly inspired by Lowe's (2006) essentialist line. Such a hybrid view bears some resemblance with other recent accounts, such as Maudlin (2007), Roberts (2008), Hendry and Rowbottom (2009), and Lange (2009, 20I I).

I do not have the space to discuss other hybrid views in detail; instead, I will focus on offering further support for one version of the hybrid view. Of the mentioned views, I believe that Hendry and Rowbottom (2009) come closest to what I have in mind. ${ }^{7}$ Their view is a type of (permissive) dispositional essentialism. While I agree with their starting point, namely that anti-quidditism does not entail nomic necessitation (p. 669), I am more doubtful about a key element in their positive proposal. This element is the (primitive) similarity between sets of dispositions - or dispositional profiles of properties. According to quidditism, the identity of properties is brute: mass and charge, say, could exchange all their dispositional features without losing their identity; they have something like a haecceity which secures their identity regardless of their dispositional features. Hendry and Rowbottom, like many others, regard this view to be incredible. Instead, they take the complete dispositional profile of any given property to be vague (p. 674). The question is, just how vague can the dispositional profile be? If no answer is given, the view runs the risk of having to return to some type of quidditism after all — not of individual dispositions, but of the dispositional profiles themselves.

While I am not fully convinced by the line of argument from Hendry and Rowbottom, there is an important connection between our accounts. The view that I will present could perhaps be understood as a type of 'weak' dispositional essentialism, as opposed to the 'permissive' type due to Hendry and Rowbottom as well as the 'strict' type that Ellis, among others, defends. Permissive dispositional essentialism is anti-quidditist: a property's identity is determined by its dispositional profile or causal role. But it is also supposed to be

\footnotetext{
${ }^{6}$ Swoyer (I982: 2II) is also hostile towards such 'intermediate' positions. Roberts (2010), on the other hand, argues that the scientific/dispositional essentialists are stuck with an 'untidy' mixed view because at least some laws turn out to be contingent.

${ }^{7}$ Thanks to an anonymous referee for The Philosophical Quarterly for inviting me to explore the connection to Hendry and Rowbottom in more detail.
} 
compatible with 'moderate' interworld variation in a property's dispositional profile. One important difference between permissive dispositional essentialism and weak dispositional essentialism of the type that I am about to develop is that whereas the former emerges simply from the idea that there could be slight variation in the dispositional profiles of properties, the latter goes some way towards explaining what might enable this variation. Keinänen (201 I), in contrasting Ellis's view with that of Hendry and Rowbottom, makes a similar observation, suggesting that 'The best way to develop 'a weak dispositionalist style' variant of Ellis's position is to assume that the exact values of certain natural constants (e.g., the gravitational constant) are contingent' (p. 423, fn. 9). As it turns out, this is exactly the strategy that I will follow, albeit focusing on the contingency of the fine structure constant rather than the gravitational constant.

Partly for dialectical reasons and partly for reasons that I have explored elsewhere (Tahko forthcoming), I will also adopt a view which is a core commitment for Lowe: natural kind fundamentalism, i.e., the thesis that there are genuine natural kinds and they form a fundamental ontological category (see Bird and Tobin 2012: section I.3). This type of view is defended both by Lowe and Ellis, and it would be possible to develop my argument from Ellis's point of view as well. Moreover, note that this assumption is not in fact necessary for the task of developing a hybrid view about laws. My choice to adopt it is largely based on personal preference as I consider the connection between laws and kinds to be an important one. In any case, the view contrasts with Armstrong's approach, which does not posit a distinct category (of universal) for natural kinds, even though Armstrong thinks that kinds 'mark true joints in nature' (1997: 67). So, the view being developed here is sympathetic to natural kind fundamentalism as developed by Lowe and Ellis, but it differs from their versions in some important respects (which will become clear below). To sum up, the two primary claims that will be put forward are as follows.

I. There is a middle ground between the two extreme views about the modal status of laws: a hybrid view, according to which some laws are contingent and some laws are necessary.

2. The distinction between laws that feature fundamental natural kinds and laws that do not feature kinds reflects the distinction between metaphysically necessary and metaphysically contingent laws.

The primary contribution of the paper concerns (2). As has been noted, various hybrid views about laws have been developed in the literature, but for the most part, I do not share the motivation behind these views. At any rate, there is more to be said about what explains the different modal status of laws. The next section examines exactly this - the apparent modal force presupposed by the initial question of the paper (MF). Some illustrative cases that may give support 
to (I) will also be discussed. In Section III, a critical assessment of Lowe's essentialist approach and the connection between laws and natural kinds are provided. A solution to some of the problems regarding Lowe's account is suggested, building on (2). The Sections IV and V concern potential examples of contingent and necessary laws in light of physics, respectively - both (I) and (2) will receive further support in these sections. A concluding section follows.

\section{THE APPARENT MODAL FORCE OF LAWS}

The initial question of this paper (MF) presupposes an apparent modal force that distinguishes genuine laws from mere regularities. As has been noted, it is not easy to make progress regarding this distinction. But something ought to be said about the source of the modal force of laws. Since I have proposed to adopt a type of essentialist view at least for the sake of argument, we can start with the idea that there are certain entities, the natures of which give rise to the causal powers that these entities manifest. In this type of account, one might suggest that it is essential that two particles with opposite charges attract each other. This regularity, according to the essentialist view, will hold in all metaphysically possible worlds. But this does not yet answer the original question, i.e., what is the source of the modal force of laws. Importantly, it seems that here we can make a distinction between what holds in virtue of the essences of, say, charged particles and what makes it the case that the law(s) governing charged particles hold across all metaphysically possible worlds - if they indeed do. In other words, the essentialist story about fundamental properties such as charge that may explain certain regularities holding across metaphysically possible worlds is distinguishable from the claim that the more general laws governing the behaviour of charged particles hold across metaphysically possible worlds. We could agree that certain regularities hold across metaphysically possible worlds, for instance, particles with like charges repel each other. However, it is making an additional commitment to say that the essentialist story about charge makes it the case that all laws governing charged particles hold in the same worlds. For instance, perhaps there is room for variation in the magnitude of the electrostatic interaction, which would also entail changes for other regularities (such as bonding behaviour). ${ }^{8}$

As a test case, take the Pauli Exclusion Principle (PEP), which states that no two fermions in a closed system can occupy the same quantum state at the same time. For example, two distinct electrons in an atom cannot have all the same quantum numbers at the same time. The PEP has numerous important implications. One of them is said to pertain to the space-occupying behaviour

\footnotetext{
${ }^{8}$ I owe a great debt to an anonymous referee for The Philosophical Quarterly for helping me formulate this idea more clearly.
} 
of all matter (we will return to this). But let's first consider a simpler case, namely the forming of ionic bonds, such as the bond between sodium and chlorine in sodium chloride molecules. The PEP illustrates the important role of the balance in the repulsion and attraction between $\mathrm{Na}^{+}$and $\mathrm{Cl}^{-}$ions. As the ions come closer - as required by the regularity of opposite charges attracting - and the wavefunctions of their electrons overlap, the PEP further requires that these electrons cannot occupy the same quantum state. The situation is resolved by a change in the energy levels of the electrons so that no two identical electrons occupy the same quantum state. This results in Pauli repulsion, which prevents the ions from coming any closer together. The product is a stable sodium chloride molecule.

The PEP expresses a regularity which appears to be central to the behaviour of all material objects; it underlies the ability of molecules to form bonds and subatomic particles to form atoms. Typically, when we consider something like the ability of sodium and chloride ions to form bonds, we state the highest order law that seems relevant. In this case, the most relevant law would seem to be Coulomb's law, which states that like charges repel and unlike charges attract. ${ }^{9}$ Since $\mathrm{Na}^{+}$and $\mathrm{Cl}^{-}$ions have opposite polarity, they are apt to bond and form sodium chloride molecules. With regard to the PEP and Coulomb's law, it may be noted that the former is rather more general than the latter.

Coulomb's law is a popular example in the literature, but the PEP less so. For instance, Bird (2007: I99) is rather hesitant about the PEP, suggesting that since Pauli's original conviction was that the principle must have an explanation within quantum mechanics, it does not state a relationship that is 'close to' fundamental, which Bird takes to be a necessary condition for laws. But as we will see, there are reasons to think that the PEP does state a relationship that is 'close to' fundamental, even if it can be given a further explanation in quantum mechanics - the 'fundamentality' of a given relationship is a rather arbitrary notion in this discussion. What we will turn to now is the suggestion that fundamental natural kinds are central to the modal force of laws.

\section{LAWS AND KINDS}

The suggestion that fundamental natural kinds are central to the modal force of laws is the core of E.J. Lowe's account of laws, which partly motivates the view being developed in this paper. Lowe suggests that if we abandon

\footnotetext{
${ }^{9}$ Following Mumford (2004: 138), one might think that there is a difference in the status of the PEP and Coloumb's law since the former is not called a law whereas the latter is - considerations like this and the fact that science does not offer us a conclusive list of laws lead Mumford (2004: ch. 8) to conclude that laws do not form a natural kind (in Ellis's sense). However, as Bird (2007: I98) has pointed out, that something is not called a 'law' does not mean that it is not a law of nature.
} 
the categoricalist view, we can offer a more satisfactory explanation of the modal force of laws. Lowe's strategy is based on his four-category ontologythe correctness of which I wish to remain neutral about. But there are some aspects of his account that are helpful for the view being developed here. Here is an illustrative passage:

What $I$ can say $[\ldots]$ is that the uniformities in question [concerning the powers and liabilities of electrons] are explained by the fact that electrons are all particular instances of the same fundamental natural kind, which is governed by a number of laws linking this kind with certain attributes. And I can explain the absence of other regularly occurring combinations of powers and liabilities in terms of the non-existence of any kinds of particle governed by suitable laws. (Lowe 2006: I6r)

Lowe proposes that we should explain laws in terms of the natures of natural kinds. For instance, it is supposedly a part of the nature of the kind 'electron' that its instances have unit negative charge. This particular essentialist fact (partly) explains certain regularities, such as the net negative charge of chloride ions. Similarly, it is a part of the nature of the kind 'fermions' that two fermions in a closed system cannot occupy the same quantum state at the same time, as the PEP states. This analysis also applies to the famous example about the universal lack of solid spheres of gold or uranium of a mile in diameter. According to Lowe (2006: I57), the case of uranium constitutes a law because it stems from the nature of the element uranium, whereas there is nothing in the nature of the element gold that would rule out solid spheres of gold a mile in diameter.

Promising as Lowe's line may seem, Bird (2012: 97-9) has recently challenged this type of approach to laws, arguing that Lowe's analysis breaks down in cases such as Coulomb's law, which does not seem to feature any fundamental natural kinds. ${ }^{10}$ The problem is that if all laws are explained in terms of kinds, then it seems that Lowe would have to postulate a natural kind which is governed by Coulomb's law. Since Coulomb's law applies to all material objects, the kind in question would have to be all-encompassing — a peculiar kind indeed. ${ }^{11}$ Bird's

${ }^{10}$ This usage of 'fundamental' should not be confused with a fundamental ontological category. Lowe (2006: 8) defines a fundamental ontological category as follows: 'the existence and identity conditions of entities belonging to that category cannot be exhaustively specified in terms of ontological dependency relations between those entities and entities belonging to other categories'. Recall that, for Lowe, natural kinds constitute one of the four fundamental ontological categories. But what Lowe seems to have in mind when he talks about 'fundamental natural kinds' in this connection is 'fundamental' in the sense of 'elementary' or 'basic', e.g., electrons are thought to be fundamental particles and hence a fundamental (basic, simple) natural kind (Lowe 2006: I54).

${ }^{11}$ Admittedly, scientific essentialists, like Ellis, might argue that Coulomb's law involves higher order determinable natural kinds, even if no fundamental natural kinds. However, this reply would not seem to be available to Lowe, who avoids the determinate-determinable distinction and states that: 'The form of a law, in the simplest case, is just this, on my view: substantial kind $K$ is characterized by Fness, or, even more simply, $K$ is $F^{\prime}$ (p. 132). For Lowe, a metaphysically necessary law must feature a fundamental natural kind, and Coulomb's law does not appear to 
central point is that some laws do not seem to feature fundamental natural kinds, and hence Lowe's analysis cannot apply to them.

A potential line of reply for Lowe would be to suggest that there is, after all, a perfectly familiar type of kind that Coulomb's law can be associated with, namely, material body. ${ }^{12}$ Even though 'material body' would be a rather general kind, it is certainly a very familiar one: Newton's laws of motion explicitly refer to such bodies exerting forces and being subjected to forces. But even if we were to accept that 'material body' is a natural kind, there are still more general laws that do not seem to feature any kinds, such as the conservation laws. Unless we are willing to go out on a limb and insist that physical systems, quite generally, constitute a natural kind, then it appears that the connection between kinds and laws required by Lowe is not forthcoming.

But perhaps there is a simple explanation for the apparent disjointness of laws and kinds. Here is one possibility: the distinction between laws that feature fundamental natural kinds and laws that do not feature kinds reflects the distinction between metaphysically necessary and metaphysically contingent lawes. Lowe himself has not entertained this type of reply, and it is doubtful that he would find it appealing. There are at least two reasons why this reply conflicts with Lowe's view. First, it entails that if there are only very few metaphysically necessary laws, then it may be possible to account for those laws with the help of only very few genuine natural kinds, contrary to what Lowe seems to think. ${ }^{13}$ Secondly, and more importantly, Lowe leaves room for metaphysically contingent laws which $d o$ feature natural kinds. Nevertheless, the line is worth pursuing for it enables us to salvage what is perhaps the most fruitful aspect of Lowe's account, namely, distinguishing between contingent law-like regularities and metaphysically necessary laws. There may be alternative solutions available for the scientific/dispositional essentialist, but the scientific/dispositional essentialist will, in turn, have difficulties accommodating the apparent contingency of some laws, as will be argued in the next section.

Why should we think that laws could come in two different varieties in the first place? Can we attribute some modal force to contingent, law-like regularities? The answers to these questions reflect the distinction between nomological (or physical/natural) and metaphysical modality. As we will shortly see, there are good reasons to think that some of the best candidates for metaphysically necessary laws do indeed feature natural kinds. But it would be odd to deny the status of law to something like Coloumb's law, which certainly expresses an

satisfy this requirement - this will be discussed in more detail below. On this, see also Wilson's (2012) recent paper, which defends the existence of fundamental determinables.

${ }^{12}$ Lowe has suggested this reply in discussion.

${ }^{13}$ By 'genuine' natural kinds, I mean mind-independent or 'joint-carving' rather than conventional kinds. See also Lowe (2009: chs 9 and I2). However, the view being developed here does not entail that there must be only very few natural kinds - this is an upshot that I have examined elsewhere (Tahko forthcoming), based on issues concerning chemical kinds in particular. 
important regularity in nature. Admittedly, this explanation calls for a further analysis of our epistemic access to the natures of kinds as well as metaphysically necessary laws - a task which I cannot hope to fully accomplish here. But even if these essentialist facts were to be considered brute facts, this view has its advantages over competitors (see Lowe 2006: I6I). However, at this point it should be stressed that the type of view being developed here does not necessarily require a commitment to (Lowe-type) natural kind fundamentalism. It would be possible to develop a hybrid approach very similar to the one proposed here strictly in terms of, say, determinable magnitudes instead of fundamental natural kinds. For Lowe, it is important that we distinguish between fundamental determinable magnitudes (such as mass and charge) and fundamental natural kinds, but the former might make for a less scientifically controversial view as physics clearly deals with fundamental determinable magnitudes, but not so obviously with fundamental natural kinds. Nevertheless, here I will continue to develop the view based on natural kind fundamentalism, as this is an additional commitment that I am willing to make (and defend). ${ }^{14}$

According to the proposed view, the apparent modal force of laws can be explained as follows. Some laws are metaphysically necessary because they feature natural kinds, but others are metaphysically contingent, law-like regularities, much like the Humean line suggests. The latter can also be said to have modal force, but only in the 'soft' sense that Bird attributes to the nomic necessitation approach, as opposed to the 'hard' sense of metaphysical modality (Bird 2005: I48). In fact, part of the reason why each of the competing views about laws has some appeal may be that typically the examples discussed by scientific/dispositional essentialists involve laws that are somewhat plausible candidates for metaphysically necessary laws, whereas the Humean view may be supported by citing cases where it appears to be relatively straightforward to come up with regularities different from the actual ones.

This sudden, even if only partial, agreement with the Humean may seem odd given that this paper started out with an explicit commitment to essentialism. The reason for this move is that even though the Humean may struggle to explain the 'hard' modal force of metaphysically necessary laws - of which there could be very few - it is enough, in the case of metaphysically contingent yet nomologically necessary laws, to explain the 'soft' modal force. Or, at any rate, there is an analysis in the vicinity of the Humean Best System Analysis which is capable of explaining nomological necessity in terms of natural properties rather than natural kinds. The appropriate distinction of laws and mere law-like regularities is hence tripartite:

I. Metaphysically necessary laws that feature fundamental natural kinds.

${ }^{14}$ For further discussion regarding natural kinds, see Tahko (forthcoming). I owe this insight to an anonymous referee for The Philosophical Quarterly. 
2. Nomologically necessary but metaphysically contingent laws that do not feature natural kinds (perhaps they feature natural properties instead).

3. Mere accidental, metaphysically and nomologically contingent regularities (not genuine laws).

Are there any reasons to think that the proposed 'hybrid' view is correct? Consider, once again, the problematic status of Coulomb's law. Bird (200I, 2002) has discussed it extensively in connection to his attempt to defend the view that (all) laws are metaphysically necessary:

Given the existence of salt and water, Coulomb's law of electrostatic attraction is sufficient to make the former dissolve in the latter. So any possible world in which salt failed to dissolve in water would be one in which Coulomb's law is false. However, it is also the case that the existence of salt depends on Coulomb's law. If Coulomb's law is false then salt cannot exist. So there is no possible world in which salt exists and in which it does not dissolve in water. (Bird 2002: 257)

On the face of it, Coulomb's law may appear to be a fairly good candidate for a metaphysically necessary law, but as we have seen, it is not clear how it could even be understood as a law from Lowe's point of view. On the other hand, Beebee (2002) has challenged Bird's analysis of Coulomb's law, defending the Humean view according to which it must be contingent: ${ }^{15}$

The fault in Bird's argument is the assumption that other possible worlds work in a nice, orderly way. In particular, the argument assumes that for a law like Coulomb's law to be false at a world $w_{\mathrm{i}}$, it has to be false in a nice, consistent, regular way. (Beebee 2002: 254)

Beebee concludes, correctly it seems, that we can introduce some peculiar, disjunctive 'law' which enables the existence of salt in a world, but where Coulomb's law is false. According to the view being developed here, this is exactly because Coulomb's law does not feature a fundamental natural kind. In the following section, an attempt will be made to demonstrate the metaphysical contingency of some laws of type (2).

\section{GONTINGENT LAWS}

In order to motivate the proposed 'hybrid' view, we ought to find plausible cases of metaphysically contingent yet nomologically necessary laws on one hand, and metaphysically necessary laws on the other hand. Let us first examine whether some laws could be contingent. ${ }^{16}$ Beebee's case for the contingency of Coulomb's law notwithstanding, I believe that the best case for the contingency

\footnotetext{
${ }^{15}$ Where $w_{i}$ is a world in which electrostatic attraction is, "necessarily, the force that exists $\left(\right.$ at $\left.w_{i}\right)$ between charged objects in virtue of some law or other that takes values of charges and separation as input and $F$ as output' (Beebee 2002: 253).

${ }^{16}$ See also Lowe's (2006: I69-7I) case for the contingency of some laws.
} 
of laws will take advantage of empirical considerations. Recent discussion concerning fundamental physical constants and their possible variation over time is of interest in this regard.

It has been suggested that at least some of the fundamental physical constants may vary over time (and even over space). Consider the fine structure constant (sometimes called the electromagnetic force coupling constant), $\alpha$, which characterizes the strength of the electromagnetic interaction, and the electron to proton mass ratio. Recent astrophysical observations of quasars appear to point towards variation of $\alpha$ over time. ${ }^{17}$ However, there are some experimental limitations in determining the variation of fundamental constants. In particular, if all the constants were to vary together so that their ratios would remain the same, it would presumably be impossible to establish this observationally. In any case, the case for $\alpha$ as a constant does not seem as firm as we once thought.

Note that the fine structure constant is a dimensionless constant which is expressed in terms of other physical constants, namely the elementary charge, the electric constant, the Planck constant, and the speed of light. The numerical value of $\alpha^{-1}$ is just over I37. A change in a dimensionless constant implies that the proportions between constants have changed. There have been attempts to explain the change in the value of $\alpha$ in terms of a change in the value of the electric constant, but it has also been suggested that the speed of light could vary. ${ }^{18}$ Even a fairly modest change in a constant like $\alpha$ would have dramatic implications. The value of $\alpha^{-1}$ must lie somewhere between i8o and 85, or else protons will decay too rapidly, and the unification of fundamental forces (omitting gravitation of course, since it has not been unified with the other forces as of yet) will not be possible (Barrow 200I: 147).

So it seems that $\alpha$ may vary over time, but what of it? Well, I would suggest that actual variation over time constitutes at least prima facie evidence for variation over metaphysically possible worlds. That is, given the actual variation of $\alpha$ over time, it does not seem to be unreasonable to imagine, say, that $\alpha$ could've had a different initial value and perhaps also a different rate of change over time. If this is the case, then it would appear to be easy to imagine that the laws of nature that involve the fine structure constant could have been different - these include all laws that concern quantum electrodynamics and indeed the example we have already discussed, Coulomb's law. Importantly, quantum electrodynamics applies to all electromagnetic phenomena associated with charged fundamental particles. Accordingly, the objection familiar from Bird (2012) could once again be applied, since a natural kind

\footnotetext{
${ }^{17}$ See Uzan (2003), and more recently Webb et al. (2011). There is still some debate about these results, but it seems that the variation of $\alpha$ over time is at least one possible explanation for the recent empirical results.

${ }^{18}$ See Kragh (2003) for some historical details.
} 
encompassing all charged fundamental particles would not seem to fit the Lowe-inspired ontology of natural kind fundamentalism that we have been working with. Yet, it seems unreasonable to deny the status of law for Coulomb's law, and indeed all laws involving quantum electrodynamics, just because they do not involve natural kinds. These laws clearly capture important, presumably nomologically necessary regularities in nature, and the Humean analysis of such laws appears plausible in the face of empirical data.

But is actual variation of $\alpha$ over time sufficient to establish variation of laws over possible worlds? One could at least object that simply the fact that $\alpha$ could have had a different value is not sufficient to establish that the laws of nature that it influences could have been different for the laws could be such that they allow for certain limited variation of $\alpha$, perhaps roughly within the values mentioned above, i.e., between I/I80 and I/85. Perhaps the fine structure constant could be tuned in such a manner that it must fall within a specific range in all metaphysically possible worlds, and hence the variation of $\alpha$ over time is part of what is in fact metaphysically necessary about the laws that it influences. A related line of thought has been explored by Marc Lange, who suggests that rather than being evidence for a 'temporary law', this type of variation over time is evidence for an 'eternal (albeit time dependent) law' (Lange 2008: 88). However, as Lange readily admits (p. 90, fn. I8), his argument for the immutability of laws is based on a specific analysis of the laws' relation to counterfactuals, which will not be acceptable to everyone (e.g., Lewis). But Lange's analysis is very much to the point given the present discussion: he argues that both in a Lewisian and in an Armstrongian analysis of laws, the immutability of laws over time must be brought in as an external element - if it is desired in the first place. Yet, this type of solution in the case at hand, it seems, would be based on a previous (ad hoc) commitment to the metaphysical necessity of the laws that $\alpha$ influences rather than any independent restrictions on the value of $\alpha$. The burden of proof, I would insist, is on the necessitarian about laws.

There are further complications. One of them concerns the typical but problematic assumption that individual laws could vary across metaphysically possible worlds. Lange's analysis, which treats laws as a system, takes this into account: 'The laws derive their lawhood collectively; their sub-nomic stability means that they are together as resilient under sub-nomic counterfactuals suppositions as they could together be. They form a unified, integrated whole - a system' (Lange 20Ir: 66). This is one reason why I am here focusing on a particular physical constant rather than a particular law - interfering with the fine structure constant will require changes throughout the system. The idea is that we should consider an alternative system entirely when dealing with the variation of laws over possible worlds. For Lange, this gives rise to 'different grades of natural necessity', which he contrasts with the flat picture defended 
by Ellis and Bird that associates a strong type of necessity (i.e., metaphysical necessity) with all laws (p. 7I). In contrast, the view being developed in this paper suggests that there are indeed different grades of necessity at play, but the crucial division is between natural and metaphysical necessity.

Let me recap the thought. If we have evidence of the time dependence of some law, $l$ (let us assume that $l$ features the fine structure constant), then this constitutes at least a prima facie reason for thinking that $l$ is metaphysically contingent. It does not guarantee that $l$ is contingent - there is still room for $l$ (as well as other laws in which constants such as $\alpha$ figure) to be structured in such a way that its time dependence is metaphysically necessary, that is, happens at the exact same rate in all metaphysically possible worlds. But the phenomenon of time dependence makes it exceedingly easy to conceive of scenarios where the rate of change is slightly different, where the initial value of $\alpha$ is slightly different, or indeed where $\alpha$ is truly constant (as physicists thought, until recently). I do not think that conceivability entails metaphysical possibility, but in this case the leap is not particularly drastic. Moreover, one might think that a time dependent law is not a law at all, but I believe that this is a mistake: What if all laws turn out to be time dependent? Even in that case, we would likely wish to salvage a sense of lawhood-perhaps it is simply overall theoretical consistency that constitutes a reason to consider time dependent laws to be laws in the first place. Yet, such constraints do not obviously reach other possible worlds. So, it is at least arguable that laws in other possible worlds are more sensitive to variation over time and hence not obviously subject the same laws at all. ${ }^{19}$

The upshot of this line of thought reflects Lange's (201r: 72) reaction towards the dispositional/scientific essentialist's case for the metaphysical necessity of laws. We may ask: Why assume that all we need to explain the metaphysical necessity of laws governing the behaviour of charged particles is that it's essential that two particles with opposite charges attract each other? In other words, even if the essentialist story is correct, it takes more to show that the relevant essences are capable of securing the necessity of the laws in which they feature. Moreover, unless there is more to the dispositional/scientific essentialist's story, there is nothing in principle that prevents someone with Humean sympathies from thinking that some of the regularities we observe are metaphysically contingent, even if there were certain more 'privileged' laws featuring fundamental natural kinds that function more or less like the essentialist account suggests.

\footnotetext{
${ }^{19}$ Thanks to Matti Eklund and Henrik Rydéhn for discussion on this point. As various commentators have pointed out, this argument is hardly conclusive. I certainly acknowledge this, and regret that I have little more to say in its defence at this time. The status of time dependent laws with regard to variation over possible worlds is an interesting area for further research.
} 
As an attempted rejoinder, the necessitarian about laws might suggest that the less privileged, metaphysically contingent laws must be grounded in some more fundamental, necessary laws and hence more fundamental natural kinds. Yet, the examples that we have been discussing based on the fine structure constant concern some of the seemingly most fundamental laws that we know of, such as the notorious Coulomb's law. The electric constant, $\epsilon_{0}$, which features in Coulomb's law, is as good a candidate for a fundamental physical constant as any, so it is difficult to see how the necessitarian could explain the variation of $\alpha$ unless some new physics helps to ground it in even more fundamental laws and kinds.

On the basis of the previous discussion, I take it that we have a fairly strong case against the view that all laws are metaphysically necessary. In fact, even the case for the necessity of any laws seems to face a serious obstacle, as the fine structure constant is crucial for a great number of supposed laws. One potential resolution is that nomologically necessary laws can take the form of boundary conditions for given constants, as was suggested above in terms of the fine structure constant. However, this is unlikely to be sufficient for 'hard' (metaphysically necessary) modal force because it appears that there could be metaphysically possible worlds where the same constants have radically different values and simply do not give rise to the same laws. But as has been suggested, those laws that feature natural kinds may fare better.

\section{NECESSARY LAWS}

The view being outlined here is sympathetic to certain aspects of Lowe's analysis of laws, specifically the idea that laws may be best understood as facts about the natures of fundamental natural kinds, where 'nature' refers to the attributes of a kind of thing (see Lowe 2006: I73). However, where the view differs from Lowe's is in claiming that not all laws must feature natural kinds. Indeed, it is consistent with (although not entailed by) the view that only very few laws feature natural kinds. The laws that do feature natural kinds have a privileged status: they are metaphysically necessary. This idea, combined with the previous case against the metaphysical necessity of some or even most laws, suggests that the attributes of a given kind of thing may only rarely be essential to the kind, especially in complex cases. Lowe himself considers the law that 'electrons have a unit negative charge' a plausible candidate for a necessary law, since it involves an elementary particle. In general, his idea seems to be that the simpler the kind in question, the more likely it is that all or most of its attributes are essential to it. This strategy is hardly conclusive without a full theory of natural kinds, but constructing such a theory is beyond the scope of the present paper (but see Tahko forthcoming). However, those who are 
sceptical about the existence of any genuine natural kinds may consider the claim to be conditional. That is, the case for metaphysically necessary laws becomes conditional on the existence of genuine natural kinds:

(COND-MET) If there are genuine natural kinds, any law which features only such kinds is metaphysically necessary. Furthermore, only those laws which feature genuine kinds are metaphysically necessary.

It will not be possible to offer a conclusive case in favour of (COND-MET) here. What can be offered is a further case study, similar to the one concerning contingent laws. These case studies are neither sufficient to establish that laws that involve genuine natural kinds are always metaphysically necessary nor sufficient to establish that laws that do not involve genuine natural kinds are never metaphysically necessary. But what they do establish is that some sort of a hybrid view is likely to be correct. (COND-MET) is a speculative explanation regarding the ontological basis of this hybrid view.

The case study in favour of the metaphysical necessity of laws is already familiar to us: the PEP and fermions - which are featured in the principlemake for a plausible candidate. ${ }^{20}$ Recall what the PEP states: if we have two identical and indistinguishable electrons (or other fermions) in a closed system, the wavefunction for the system of those two electrons must be antisymmetric. Electrons occupy successively higher orbitals to prevent a shared quantum state, and hence not all electrons can collapse to the lowest orbital. It is a property of any closed system, such as an atom, that any two electrons in that closed system do not have the same set of the four quantum numbers. When the conception of laws at hand is applied to this case, a plausible way to understand the PEP is in terms of the attributes of electrons and other fermions. In particular, it is part of the nature of fermions that they behave in a manner that is constrained by the PEP. This may be the result of a combination of the modal constraints that particular attributes of fermions - such as their half-integer spin - have. The role of spin is highlighted by the fact that particles of half-integer spin must have anti-symmetric wavefunctions, whereas particles of integer spin must have symmetric wavefunctions. The latter, bosons, are not subject to the PEP. ${ }^{21}$

Clearly, the fact that the behaviour of fermions is constrained by the PEP is at least partly due to their half-integer spin, given that in the view under consideration, metaphysically necessary laws are simply facts about the natures of natural kinds. It is also plausible that half-integer spin is essential for fermions, since it is what differentiates them from bosons; the behaviour of

\footnotetext{
${ }^{20}$ Here the focus is on reasons for thinking that PEP is metaphysically necessary. For further evidence on why it must feature a genuine natural kind, see Tahko (2012).

${ }^{21}$ It is, however, not implausible that bosons as well constitute a genuine natural kind. Accordingly, there may be different metaphysically necessary laws featuring bosons.
} 
fermions and bosons is radically different, especially at low temperatures. If Lowe (2006: 169-70) is right about how simple kinds and their attributes are linked, this would suggest that both fermions and bosons have most of their attributes essentially, that is, they depend for their existence and identity on these attributes. Hence, both would be likely candidates for genuine kinds. But recall that the present argument (based on (COND-MET)) is only conditional - the real battle for natural kinds will have to take place elsewhere.

In light of the above, the PEP is one plausible candidate - the most plausible that I have been able to come up with - for a metaphysically necessary law of nature, even though I do not claim to have conclusively demonstrated this. However, even if the PEP itself did not hold by metaphysical necessity, some law very similar to it would likely be necessary as any binding behaviour, or indeed the stability of matter, would seem to require something like the PEP. Of course, if a universe with no binding behaviour is metaphysically possible, then this might serve as a counterexample - at least there would presumably be no complex kinds in such a universe. ${ }^{22}$ But that would be an odd universe indeed: to serve as a counterexample, it should contain fermions. If what was said above about PEP was correct, then these fermions would have to lack features like half-integer spin, since features like half-integer spin entail modal constraints such as the one that the PEP states. However, these are the very constraints that enable binding behaviour in the first place. To all ends and purposes, then, those odd fermions would appear to behave like - and be indistinguishable from-bosons. A universe with no binding behaviour is plausibly a universe with no fermions, and a universe with no fermions is not a valid counterexample to the metaphysical necessity of the PEP, since it does not contain the natural kind featured in the PEP. ${ }^{23}$ This corroborates (GOND-MET).

Of course, there is an obvious caveat here: the argument constructed above assumes anti-quidditism - the view that the identity of properties is not bruteat least in the case of fermions. If there are metaphysically possible worlds with fermions* that are not constrained by some analogue of the PEP and hence fail to fill the causal role of the actual fermions, then only a quidditist would have the tools to identify fermions and fermions*. However, I consider the burden of proof to be strictly on the quidditist - and here I am surely with the majority (e.g., Mumford 2004: I04). Moreover, since I have adopted a type of essentialist approach in the first place, I would hope that this justifies asserting anti-quidditism in the case of fermions, even if I do acknowledge the need to

${ }^{22}$ By this I do not mean an absolutely empty universe, whether or not such a universe is metaphysically possible. An empty universe would not be a suitable counterexample because it would also lack anything that could be constrained by the PEP. More generally, if we understand laws as facts about the natures of natural kinds, then possible worlds where the kind does not exist are not viable counterexamples.

${ }^{23}$ We could perhaps say that the PEP is vacuously true in such worlds. 
defend this assumption in more detail. Anti-quidditism may beg the question against the Humean, but this should be excusable given the dialectic of the paper. In particular, if the view developed here is understood as a type of 'weak' dispositional essentialism, then it is intimately connected to anti-quidditism at the outset. As Hendry and Rowbottom put it when outlining their 'permissive' dispositional essentialism, 'if the modal intuitions concerning quiddities and counternomic situations have some pull, however weak, a position that honours them will have an advantage over both quidditism and strict dispositional essentialism' (Hendry and Rowbottom 2009: 672).

\section{GONGLUSION}

It is a theoretical advantage of the view that I have outlined that no single case can refute it. If we acknowledge that some laws are metaphysically necessary, but not all of them (and if we are fallibilist about which laws are necessary and which ones are not), then it is possible to agree with the scientific/dispositional essentialist about some cases and with various Humeans about others. We can even change our minds about specific cases as new evidence emerges. This conception about the status of laws is no doubt closer to the scientific/dispositional essentialist view, but it is truly a hybrid view, and the manner in which I have laid it out - by assuming a type of essentialist position and natural kind fundamentalism - is by no means the only possibility. To motivate this view, it was suggested that we have good reasons to think that some laws are metaphysically necessary, whereas some laws are metaphysically contingent yet nomologically necessary. This is enough to cause trouble both for the Humean about laws as well as the scientific/dispositional essentialist view - at least in their strongest form - since the former regards all laws as metaphysically contingent, whereas the latter generally regards all laws as metaphysically necessary.

Before concluding, one objection should be anticipated. If the preceding line of thought is correct, it might be argued that no reason has been given to consider metaphysically contingent laws to be lawes at all. Why shouldn't we just say that all lawes are metaphysically necessary - because they feature natural kinds - and what remains are not genuine laws, whether nomologically necessary or not? Indeed, the view at hand does suggest that there is an ontological difference between metaphysically contingent and metaphysically necessary laws. There is no reason to consider this approach to be 'untidy', in contrast to Bird, since the difference between these two classes of laws can be explained in virtue of their source. But the metaphysically contingent laws may certainly be called something else than laws if one so wishes. Perhaps better, 
we could call contingent laws weak and necessary laws strong, reflecting their modal force and applicability - I prefer these labels to Bird's 'soft' and 'hard'.

The resulting view, that some laws are metaphysically contingent and some necessary, is perhaps easier to accept for a scientific/dispositional essentialist, since the view does still hold that metaphysically necessary laws emerge from the natures or essences of kinds. Hence, even if there were only very few metaphysically necessary laws of nature, it is in fact the source of the modal constraints imposed by laws that is at issue here. Since the proponents of HS and nomic necessitation deny that there could be any immanent modality involved in laws, they have no easy way to account for the source of these modal constraints in the case of metaphysically necessary laws. The case of the PEP demonstrates that at least some laws do appear to impose modal constraints of the metaphysical sort - insofar as they feature genuine natural kinds. ${ }^{24}$

\section{REFERENCES}

Armstrong, D. M. (1983) What is a Law of Nature? Cambridge: CUP.

- (1997) A World of States of Affairs. Cambridge: CUP.

Barrow, J. D. (200I) 'Cosmology, Life, and the Anthropic Principle', Annals of the New York Academy of Sciences, 950: 139-53.

Beebee, H. (2000) 'The Non-Governing Conception of Laws of Nature', Philosophy and Phenomenological Research, 61: 57I-94.

(2002) 'Contingent Laws Rule: Reply to Bird', Analysis, 62: 252-5.

Bird, A. (200I) 'Necessarily, Salt Dissolves in Water', Analysis, 6r: 267-74. (2002) 'On Whether Some Laws Are Necessary', Analysis, 62: 257-70.

— (2005) 'The Ultimate Argument against Armstrong's Contingent Necessitation View of Laws', Analysis, 65: 147-55.

- (2007) Nature's Metaphysics. Oxford: Clarendon Press. - (2012) 'Are Any Kinds Ontologically Fundamental?', in T. E. Tahko (ed.) Contemporary Aristotelian Metaphysics, 94-I04. Cambridge: CUP.

- and Tobin, E. (2012) 'Natural Kinds', in E.N. Zalta (ed.) The Stanford Encyclopedia of Philosophy (Winter 2012 Edition). The Metaphysics Research Lab, Center for the Study of Language and Information (CSLI), Stanford University (http://plato.stanford.edu/ archives/win2012/entries/natural-kinds/> (last accessed is I5 January, 20I5).

Earman, J. and Roberts, J. T. (2005a) 'Contact with the Nomic: A Challenge for Deniers of Humean Supervenience about Laws of Nature Part I: Humean Supervenience', Philosophy and Phenomenological Research, 71: I-22.

- $(2005 \mathrm{~b})$ 'Contact with the Nomic: A Challenge for Deniers of Humean Supervenience about Laws of Nature Part Ir: The Epistemological Argument for Humean Supervenience', Philosophy and Phenomenological Research, 71: 253-86.

Ellis, B. (200I) Scientific Essentialism. Cambridge: CUP.

and Lierse, C. (1994) 'Dispositional Essentialism', Australasian fournal of Philosophy, 72: 27-45.

${ }^{24}$ I would like to thank Jason Bowers, Eyja Brynjarsdóttir, Travis Dumsday, Markku Keinänen, Markus Schrenk, and two anonymous referees for The Philosophical Quarterly for helpful comments on earlier versions of this paper. Thanks also to audiences at Uppsala, Bratislava, Oxford, Bristol, and Reading, where this paper was presented. Research for this paper was made possible by a grant from the Academy of Finland. 
Hendry, R. and Rowbottom, D. (2009) 'Dispositional Essentialism and the Necessity of Laws', Analysis, 69: 668-77.

Keinänen, M. (201 I) 'Tropes - The Basic Constituents of Powerful Particulars?', Dialectica, 65: $4^{1} 9^{-50}$.

Kragh, H. (2003) 'Magic Number: A Partial History of the Fine-Structure Constant', Archive for History of Exact Sciences, 57: 395-43I.

Lange, M. (2008) 'Could the Laws of Nature Change?', Philosophy of Science, 75: 69-92.

- (2009) Lawes and Lawmakers. Oxford: OUP.

(20I I) 'It Takes More Than All Kinds to Make a World', in J. K. Cambell, M. O'Rourke and M. H. Slater (eds) Carving Nature at Its foints. 53-84, Cambridge, MA: MIT Press.

Lewis, D. K. (1986) Philosophical Papers. vol. 2. New York, NY: OUP.

Loewer, B. (2012) 'Two Accounts of Laws and Time', Philosophical Studies, I6o: II 5-37.

Lowe, E. J. (2006) The Four-Category Ontology. Oxford: OUP. (2009) More Kinds of Being. Oxford: Wiley-Blackwell.

Maudlin, T. (2007) The Metaphysics Within Physics. Oxford: OUP.

Mumford, S. (2004) Lawes in Nature. London: Routledge.

Roberts, J. T. (2008) The Law-Governed Universe. Oxford: OUP.

(2010) 'Some Laws of Nature are Metaphysically Contingent', Australasian Fournal of Philosophy, 88: $445^{-57}$.

Schrenk, M. (20I I) 'Interfering with Nomological Necessity', The Philosophical Quarterly, 6r: 577-97

Swoyer, C. (1982) 'The Nature of Natural Laws', Australasian Fournal of Philosophy, 60: 203-23.

Tahko, T.E. (2012) 'Boundaries in Reality', Ratio, 25: 405-24.

_ (forthcoming) 'Natural Kind Essentialism Revisited', Mind.

Uzan, J.-P. (2003) 'The Fundamental Constants and Their Variation: Observational Status and Theoretical Motivations', Reviewes of Modern Physics, 75: 403-55.

Webb, J. et al. (201 I) 'Evidence for Spatial Variation of the Fine Structure Constant', Physical Review Letters, I07: I9IIоI.

Wilson, J. (2012) 'Fundamental Determinables', Philosophers' Imprint, I2: 4.

University of Helsinki, Finland 